CP, 2015, Vol.4 - Nº7, pp. 124-140. ISSN 2014-6752. Girona (Catalunya). ÁLVAREZ-ARÁMBULA, Erika Guadalupe: Uso de las aplicaciones de Internet en personas mayores en Barcelona: Un estudio desde la perspectiva de género. Recibido: 06/10/2015 - Aceptado: 26/11/2015

\title{
USO DE LAS APLICACIONES DE INTERNET EN PERSONAS MAYORES EN BARCELONA: UN ESTUDIO DESDE LA PERSPECTIVA DE GÉNERO ${ }^{1}$
}

\author{
Uses of Internet applications in elderly people in Barcelona City: \\ A study from gender perspective
}

\section{AUTORA: ÁLVAREZ-ARÁMBULA, Erika Guadalupe}

\author{
Investigadora - Universitat Autònoma de Barcelona - España
}

eriaram@yahoo.com

\section{Resumen}

El envejecimiento de la población es un fenómeno a nivel mundial que preocupa a diferentes organismos nacionales e internacionales, éstos llevan a cabo distintas acciones para mejorar la calidad de vida de las personas mayores a través del desarrollo de habilidades digitales que les permitan afrontar el cambio tecnológico. Este estudio forma parte de la tesis doctoral presentada en 2014. Es de tipo cualitativo y empírico y, pretende mostrar cómo las personas mayores afrontan el cambio tecnológico a través del uso y consumo de las aplicaciones de Internet, y cómo adquieren y desarrollan habilidades digitales. Para ello se realizaron entrevistas a profundidad a diez mujeres y diez hombres, residentes en Barcelona, con base en la tipología "media-person interactions" (McQuail, Blumler y Brown, 1972) que se desprende de la Teoría de Usos y Gratificaciones (Katz y Blumler, 1974). Además, se aplicó la perspectiva de género, lo que permitió hacer una comparación entre hombres y mujeres mayores y detectar así las diferencias y similitudes entre ambos al hacer uso de las aplicaciones de Internet. La recogida de datos se realizó durante cuatro meses, en dos periodos de dos meses cada uno: Marzo-Abril y Septiembre-Octubre de 2011.

\section{Palabras clave}

Cambio tecnológico; habilidades digitales; inclusión digital; personas mayores; perspectiva de género.

\begin{abstract}
The phenomenon of aging population is increasing around the world and this situation is grabbing attention to different national and international organizations to carry out different actions looking to improve the quality of life in elderly people through developing digital skills to face the technological shift. The following study is part of the doctoral thesis presented in 2014. This is an empirical and qualitative study and pretends to show how elderly people face the technological shift through using and consuming the Internet applications and how they obtain and develop digital abilities. We applied depth interview to ten elderly men and ten elderly women living in Barcelona City, and was structured according to "media-person interactions" typology (McQuail, Blumler and Brown, 1972) detached from the Uses and Gratifications Theory (Katz and Blumler, 1974). Also, we applied the gender perspective, allowing a comparison between elderly men and women to detect differences and similarities when they use the Internet applications. The data was gathered in four months in two periods of two months each one from March to April and September to October in 2011.
\end{abstract}

\section{Key words}

Digital inclusion; digital skills; elderly people; gender perspective; technological shift.

\section{Introducción}

El siguiente estudio surge de la inquietud por querer conocer más de cerca el fenómeno del envejecimiento de la población y su relación con el uso de Internet y sus aplicaciones. Es decir, se tratan dos fenómenos que están cambiando y que se fusionan en la transición de lo analógico a lo digital para evitar la

\footnotetext{
${ }^{1}$ Éste trabajo ha sido financiado por el Consejo Nacional de Ciencia y Tecnología - CONACYT
}

brecha digital y la brecha digital de género dentro del colectivo de personas mayores.

Los objetivos propuestos para este estudio fueron indagar en cómo hombres y mujeres mayores obtienen las diferentes habilidades para hacer uso de las aplicaciones de Internet, así como la frecuencia y duración de uso de las mismas en ambos casos. 
Podría pensarse que, las personas mayores, al pertenecer a una generación que no creció haciendo uso de la tecnología, también llamadas inmigrantes digitales (Prensky, 2001), se han tenido que adaptar a los cambios tecnológicos, y prefieren las "gratificaciones" que les proporcionan otros medios más tradicionales como la $\mathrm{TV}$, la radio, la prensa escrita o el teléfono. Y por tanto, no están interesadas en desarrollar habilidades tecnológicas.

Por lo que se propuso dar respuesta a las siguientes preguntas de investigación: ¿Qué aplicaciones de Internet usan hombres y mujeres mayores?, ¿qué tiempo usan cada una de las aplicaciones de Internet para incorporarlas a su vida cotidiana? y ¿qué diferencias existen, en cada una de las circunstancias anteriores, según el género?

Y así determinar si el uso de las aplicaciones de Internet es gratificante para hombres y mujeres mayores al cubrir algunas necesidades relacionadas con: Diversión, Relaciones personales, Identidad personal, Búsqueda de información, Acceso a otros medios (TV, radio, prensa) y desarrollo de habilidades digitales de cara al Futuro.

Considerando que la población europea de personas de 65 y más años se incrementará casi el doble, al pasar de 87.5 millones en 2010 a 152.6 millones en 2060. Y que el número de personas pertenecientes a la población de 80 y más años, se incrementará casi el triple para el mismo periodo en la Unión Europea al pasar de 23.7 millones en 2010 a 62.4 millones en 2060 (Comisión Europea. Asuntos Económicos y Financieros, 2012: 27). Es necesario dotar de habilidades digitales a este grupo social a través del aprendizaje, práctica y desarrollo de las mismas, así como de futuras habilidades para afrontar el cambio tecnológico que se vive actualmente y que continuará con el paso de los años.

Por esto, hablar del uso de Internet, es hablar de inclusión social, que tiene como objetivo la igualdad social sin importar sexo, género, edad o perfil socioeconómico. De aquí que los diferentes gobiernos se preocupen por ofrecer acceso a las Tecnologías de la Información y la Comunicación (TIC), contribuyendo a que la brecha digital o "la difusión dispar de la tec- nología y las comunicaciones" (PNUD, 2001: 40) se estreche en las diferentes sociedades.

En España, ser una persona mayor, es ser una persona que legalmente tiene derecho a ciertos beneficios sociales como las pensiones, y esto se aplica en su gran mayoría a los hombres más que a las mujeres. Ser una persona mayor, también hace referencia al deterioro de funciones psicomotrices vinculadas a la salud, que es uno de los temas más desarrollados en relación con las TIC, pues se pretende que las personas mayores sean más independientes en cuanto al cuidado y monitoreo de su salud (Giddens, 1991; Zamorano, 2004).

El recorrido realizado a través de los diferentes estudios dirigidos a personas mayores y el uso de las TIC, así como las consecuencias y/o problemas a los que se ha enfrentado este colectivo al hacer uso de la tecnología, permitió que el estudio se enriqueciera y mejorara aportando otro punto de vista.

Actualmente el interés en este tema ha generado varias investigaciones, como por ejemplo, en 2009 Mordini et al., en el que tratan el problema de soledad y aislamiento que se da en el colectivo de personas mayores al llegar a esta fase de la vida (estar lejos de sus familiares por distancias físicas, como por ejemplo, el vivir en países diferentes, en hogares diferentes, etc.), y cómo las TIC, a través de la Inclusión Digital, pueden ayudar a "sobrellevar" esta situación, ofreciendo una mejor calidad de vida y acabando con el aislamiento social.

La tesis doctoral titulada ¿dónde estás...? El teléfono móvil y la vida cotidiana. El caso de las personas mayores en la ciudad de Barcelona (Ramírez Pino, 2008), aborda la situación de las personas mayores residentes en Barcelona frente al uso del teléfono móvil, así como las consecuencias sociopolíticas y económicas que se derivan de dicho uso.

También, Anderson y Wesley (2007) de la Louisiana State University, en su estudio Circumvention and Social Change: ICTs and the Discourse of Empowerment, realizaron una investigación, desde una perspectiva feminista, centrada en el empoderamiento de las 
mujeres que buscan ocupar un lugar importante en el ámbito de las TIC.

Al respecto, Abbate (2000), afirma que la llegada de las nuevas tecnologías a sociedades más conservadoras-machistas, daría como resultado visible un colectivo de mujeres con pocos recursos y un porcentaje muy bajo en cuanto a la adopción y uso de las mismas. De aquí que varios estudios se centren en enseñar a las mujeres el uso de las TIC y tengan como objetivo crear en ellas la necesidad de fomentar el uso de las mismas.

\section{Marco Teórico}

El estudio tiene como teoría principal la Teoría de Usos y Gratificaciones (Katz y Blumler, 1974) de la que se desprende la tipología "media-person interactions" (McQuail, Blumler y Brown, 1972), con los siguientes bloques temáticos:

Diversión, que hace referencia a las formas de escape y ocio que buscan para alejarse de la rutina diaria y los problemas. Relaciones personales, que hace referencia a la forma en que se relacionan las personas mayores con sus seres queridos a través de las TIC. Identidad personal, que hace referencia a todas aquellas expresiones que identifican al usuario. $\mathrm{Y}$ Búsqueda de información, que hace referencia a los intereses de los usuarios que les motivan a buscar contenidos en línea: Tiempo de ocio, liberación emocional, excitación sexual...

Además, se agregaron dos tópicos más: Acceso a otros medios, que hace referencia a cómo el usuario accede a otros medios como TV, vídeos tipo YouTube, emisoras de radio, periódicos o diarios a través de Internet gracias a la actual convergencia de medios que permite diferentes posibilidades de uso y que requiere nuevas habilidades digitales, y Futuro, que hace referencia a las expectativas que tiene el usuario de cara al futuro como el desarrollo de nuevas habilidades digitales para satisfacer otras necesidades u obtener otras gratificaciones una vez que han incorporado las TIC a su entorno cotidiano.

\section{Método}

Este estudio es de tipo cualitativo (Strauss y Corbin, 2002: 19, 20), está dentro del paradigma constructivista con una base epsitemológica, (Guba y Lincoln, 2002: 121, 130) y un enfoque émic, (Pike, 1954: 49). Y se identifica con el determinismo social. Es decir, "La tecnología se ve influida por las necesidades individuales y los contextos sociales” (Castells, 2004).

Se ha propuesto como instrumento principal de recolección de datos la entrevista semi-estructurada a profundidad, lo que permite salirse del guión propuesto con la finalidad de obtener información relevante y de calidad para el estudio.

Como complemento a la entrevista y al término de la misma, se les entregó un diario de campo, con soporte en papel, que regresaron con datos, fruto de su propia observación y conducta, en el cual llevaron un registro de sus actividades en línea durante diez días, incluyendo fines de semana, y en el que aportaron la siguiente información: fecha de uso, tiempo de uso, dificultades a las que se enfrentaron y otros comentarios.
Una vez recolectada la información relacionada con la entrevista, se aplicó el análisis de contenido, que según Krippendorff (1990) es "la técnica destinada a formular, a partir de ciertos datos, inferencias reproducibles y válidas que puedan aplicarse a un contexto" (Krippendorff, 1990: 28). Mientras que para el diario de campo se aplicó la categoría física (Krippendorff, 1990), para ubicar la posición y duración o extensión, en este caso, del uso de una aplicación de Internet. Todos los datos fueron analizados con el programa Atlas Ti 6.1., software para el análisis de datos cualitativos.

Además, y como parte fundamental del estudio, se aplicó la perspectiva de género a todos los datos. Gracias a esta estrategia es posible detectar diferencias y similitudes entre hombres y mujeres, lo que propicia la igualdad de oportunidades entre los géneros y por lo tanto, pretende acabar con la brecha digital de género definida como una “... enorme brecha social que se sustenta en factores de exclusión como la capacidad económica, la disponibilidad de tiempo, los 


\section{Selección de la muestra}

La selección de la muestra se hizo a través del método de muestreo no probabilístico intencional. Es decir, la muestra no es aleatoria ni probabilística y fue seleccionada de acuerdo a las características que busca el investigador (Sampieri, 2007: 263).

Como sujetos de estudio se propusieron dos grupos de personas: pre-mayores, entre los 50 y 54 años, que se acercan a esta etapa de la vida con un alto interés por desarrollar habilidades digitales antes del momento de la jubilación. Y personas mayores, de 55 años en adelante, que "han envejecido con éxito" (Zamorano, 2004: 225), es decir, personas que han llegado a esta etapa de la vida en un estado funcional donde los niveles de dependencia son bajos o nulos.
Para localizar a estos sujetos se recurrió a diferentes técnicas como el "boca a boca" o el "snow ball" para establecer los primeros contactos. La estrategia fue comenzar por las generaciones más jóvenes, quienes ayudaron a encontrar a personas mayores con el perfil que necesitábamos. Más tarde, estas personas mayores sugirieron otros posibles candidatos.

Los criterios de selección fueron: ser mayor de 50 años (pre-mayores y mayores) y tener conocimientos previos sobre el uso de las aplicaciones de Internet, tener un ordenador en casa o poder acceder a uno de manera fácil y frecuente y vivir en la zona Metropolitana de Barcelona, sin importar la nacionalida.

\section{Trabajo de campo}

La recogida de datos se realizó durante cuatro meses, divididos en dos periodos de dos meses cada uno: Marzo-Abril y Septiembre-Octubre de 2011.

Se aplicaron entrevistas a profundidad a un mismo número de hombres que de mujeres: diez hombres y diez mujeres. Las entrevistas se realizaron en el domicilio de los sujetos o en cafeterías cercanas, con una duración aproximada de 20 a 35 minutos. Todos los sujetos estuvieron de acuerdo en que la entrevista fuera grabada y colaboraron de manera totalmente desinteresada.

Una vez culminada la entrevista, se les entregó el diario de campo que debía ser devuelto una vez realizado el registro de sus actividades en línea durante los diez días propuestos.

\section{Resultados}

A continuación se presentan los resultados obtenidos en el trabajo de campo. El siguiente gráfico muestra una comparación en el uso de las aplicaciones de Internet en hombres y mujeres mayores (ver gráfico 1).

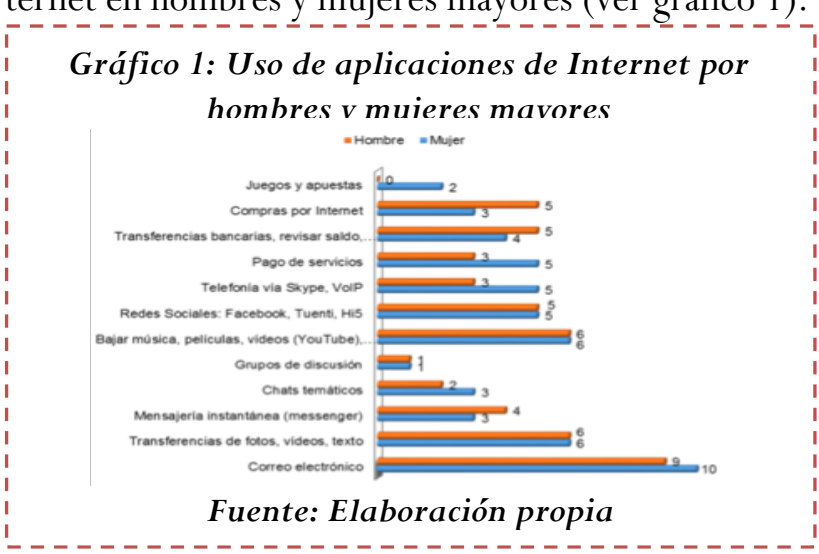

En el gráfico anterior se observa una similitud entre hombres y mujeres mayores que buscan estar en contacto con familiares, amigos y otras personas, sobre todo a través del correo electrónico que es la aplicación más popular dentro de esta generación. Tanto hombres como mujeres mayores hacen uso de las Redes Sociales, esto les ayuda a desarrollar numerosas habilidades digitales que les van a servir para el uso de nuevas aplicaciones. Además, ambos están interesados en realizar operaciones a través de Internet que normalmente se hacían cara a cara como comprar y realizar pagos de servicios.

Otra similitud es que en ambos casos, se sienten más atraídos por las aplicaciones dirigidas al ocio y entre- 
tenimiento, como bajar música, películas, vídeos, hacer transferencias de fotos, texto y vídeos, que por las aplicaciones para participar en grupos de discusión y chats temáticos.

De acuerdo con la tipología "media-person interactions" McQuail, Blumler y Brown (1972), se presentan los resultados por tópico: Diversión, Relaciones personales, Identidad personal, Búsqueda de información, Acceso a otros medios y Futuro.

\subsection{Diversión}

Los hombres utilizan las aplicaciones de Internet para pasar el tiempo y buscar información. Dedicando de dos a tres horas diarias y en el caso de personas con problemas físicos dos ó tres horas sólo algunos días de la semana.

El tiempo que dedican tanto hombres como mujeres a hacer uso de Internet varía, dependiendo de la actividad:

"Durante el día una hora, pero en la noche de dos a tres horas." (Mujer 7, 71 años)

"Un día, igual nada y otro día, igual me tiro dos horas o tres, depende." (Mujer 3, 66 años)

"Por la mañana pongo las noticias, tres cuartos de hora o una hora. Y luego, por la tarde, pues suelo estar de cinco a siete u ocho horas. Y después de cenar, unas dos o tres horas más. (Hombre 8, 63 años)

"Es que no lo uso diario, depende, treinta minutos, una hora y se acabó. Una hora ya es mucho para mí, ¿eh?” (Hombre 4, 76 años)

Las mujeres las usan para estar en contacto con familiares y amigos y evitar el aislamiento. Dedicando de una a dos horas diarias por la mañana y de dos a tres horas por la tarde varios días a la semana, ya que compaginan sus actividades diarias con el uso de las aplicaciones de Internet.

"Durante el día una hora, pero en la noche de dos a tres horas." (Mujer 7, 71 años)
"Sí, me gusta porque me distrae. Se me pasa el tiempo y no me doy cuenta." (Mujer 3, 66 años)

La mayoría de las mujeres considera que hacer uso de las aplicaciones de Internet les ayuda a salir de la rutina diaria.

"Sí, sí, sí... [...] encuentro que es lo que la vida nos trae. Es lo más interesante que hay." (Mujer 7, 71 años)

"Sí, porque de alguna manera, ya estoy cansada, estoy jubilada y estoy harta de fregar, de limpiar y de nietos y de maridos y de todo y me meto al facebook y me paso un buen rato distraída." (Mujer 8,62 años)

En el caso de los hombres hay diferentes opiniones. Unos consideran que hacer uso de Internet es parte de la rutina. Otros afirman que les permite salir de la rutina diaria porque pueden realizar diferentes actividades alternándolas con otras en las que no es necesario el ordenador.

"Mmmm... no, porque hombre, por nuestro trabajo ya es una rutina estar en Internet. No rutina, es que sin Internet estás perdido.” (Hombre 10, 61 años)

"Sí. Me permite hacer diversas actividades. Ir turnando, no estar todo el tiempo en una." (Hombre 7, 67 años)

De acuerdo con este tópico, las mujeres usan las aplicaciones de Internet para comunicarse con familiares y amigos y realizan actividades de ocio digital. Y las que pasan más tiempo en casa hablan en línea con otras personas para no sentirse aisladas, divertirse y llenar su tiempo libre con actividades distintas a las tareas domésticas. Mientras que los hombres leen el periódico para informarse de lo que acontece en el mundo, leen libros, escuchan música, etc.

\subsection{Relaciones personales}

Los hombres utilizan las aplicaciones de Internet por entretenimiento y contactan con familiares y amigos para quedar, ya que prefieren el trato personal. 
"Con empresas y otros profesores y otros de la administración. Únicamente relaciones laborales." (Hombre 3, 59 años)

"Es simplemente comercial. Y sólo con clientes o con grupo de amigos, o con grupo de fans de nuestra página...” (Hombre 10, 61 años)

"Con amistades. Para estar en contacto con ellos, y salir con ellos.” (Hombre 1, 55 años)

Mientras que las mujeres mantienen contacto en línea con su círculo más cercano y con otras persona que viven lejos.

"Con amigos, hijos, poco más, o sea, familia. Es muy cómoda, porque es lo que hay hoy en día, porque un teléfono ya no funciona." (Mujer 8, 62 años)

"Las personas con las que mantengo contacto son familiares y amigos. Es económico, sería mejor el teléfono, pero es caro, es una opción, no tan directa, pero te permite comunicarte." (Mujer 2, 52 años)

Ambos utilizan el correo electrónico, facebook o Skype. Y la mayoría de las veces realizan sus actividades solos(as).

\section{“facebook, y el correo." (Mujer 6, 70 años) \\ "Correo electrónico, Skype, el chat un poco." (Mujer 8, 62 años)}

“Correo electrónico y ya está. Bueno, a veces me pongo a chatear con mi nieta. Un poco, no mucho." (Hombre 6, 77 años)

"Normalmente el correo electrónico y el Skype. [...] practicaba el messenger con una persona que estaba en México y ya falleció.” (Hombre 8, 63 años)

Las mujeres consideran que hacer uso de Internet les aporta numerosos beneficios a nivel personal y valoran las múltiples posibilidades que éste les ofrece para relacionarse con los demás, especialmente con fami- liares y amigos. Además, sienten que les facilita el desempeño de las tareas cotidianas que ahora pueden realizar desde casa, ahorrando tiempo y esfuerzo.

"Aparte de mantener el cerebro activo,... porque se entretiene uno mucho y bueno, veo otras formas de relacionarse.” (Mujer 4, 63 años)

"Me distrae, me relaciona con gente, que si no, bueno, tendría que escribir o telefonear." (Mujer 5, 81 años)

Los hombres, por su parte, también encuentran beneficioso el uso de las aplicaciones de Internet, aunque no tanto como las mujeres. Lo utilizan más bien para la búsqueda de información sobre temas concretos relacionados con sus hobbies, entretenimiento, noticias, trabajo...

"Porque es una herramienta más de nuestro trabajo, es un canal y es una ventana para exponer nuestros productos." (Hombre 10, 61 años)

"Enormemente. Me permite hacer cosas que antes no. Primero agilidad y luego contacto muy directo. Resolver problemas en poco tiempo..." (Hombre 7, 67 años)

El uso de las aplicaciones de Internet en hombres y mujeres mayores no influye en el incremento de amistades pero sí en una mayor interacción con personas ya conocidas.

"No. porque no me abro hacia el exterior, siempre son los mismos..." (Mujer 8, 62 años)

"No, porque las amistades generalmente las hago personalmente...” (Mujer 2, 52 años)

"De amistades no, en todo caso, que algunos viejos amigos nos volvemos a encontrar." (Hombre 7, 67 años)

"No. Ha podido aumentar [...] la frecuencia con alguna persona, pero aumentar (el número), no.” (Hombre 8, 63 años) 
Referente a este tópico, Relaciones personales, las personas mayores, en general, desconfían de aquellas personas que buscan contactar con ellos en línea, sin haberse conocido previamente (cara a cara). En consecuencia, el uso de las aplicaciones para comunicarse con otras personas, no ha contribuido a aumentar el número de sus amistades, pero sí la frecuencia con la que establecen contacto con sus familiares, amigos y personas cercanas a su entorno.

\subsection{Identidad personal}

La mayoría de los hombres se identifica con personas de su misma edad y de su círculo más cercano, incluyendo compañeros de trabajo.

"No, aunque es probable que mis amigos sean más próximos a mi edad que a otra. Pero también tengo hijos, pues también." (Hombre 2, 65 años)

"Mayormente sí, gente de la misma edad o más mayor porque con una persona joven no te puedes meter, ¿sabes?, porque ni te hace caso...” (Hombre 5, 81 años)

No usan las redes sociales porque no les interesa, les parece peligroso exponer tanta información y consideran que son para jóvenes. Sin embargo sí usan facebook y LinkedIn si es con fines comerciales o profesionales.

"No, $[\ldots]$ es un factor incontrolable, $[\ldots]$ en el momento en el que pongas cosas tuyas ya no vas a ser dueño de tu destino..." (Hombre 8, 63 años)

"No. Meterme en un foro y ponerme a discutir, no. $\mathrm{Y}$ creo que estoy un poco fuera de eso. Eso es para gente más joven." (Hombre 9, 61 años)

"LinkedIn [...] la que utilizamos en la empresa para conectar con nuestros clientes actuales y potenciales.” (Hombre 7, 67 años)

"Sí. Mis razones son meramente comerciales." (Hombre 10, 61 años)
Las mujeres por el contrario, se identifican con personas que comparten sus mismos intereses sin importar la edad. Su motivación para usar las redes sociales (facebook) es estar al tanto de lo que sucede a familiares y amigos. El uso de las redes sociales supone un reto para ellas debido a que generaciones más jóvenes las utilizan.

"Sí, facebook. Y quiero aprender a usar Twitter." (Mujer 10, 89 años)

"Pues que alguien como yo, está haciendo cosas de gente joven y eso me da seguridad y que estoy y pertenezco al mundo de hoy, de ahora mismo." (Mujer 4, 63 años)

"La comunicación, me gusta para no terminar, si Dios quiere, con Alzheimer, pues, pues tener un poco la mente, aprender cosas nuevas, me gusta para desarrollar un poco y para las pocas neuronas que nos quedan.” (Mujer 9, 64 años)

En cuanto a Identidad personal, los hombres usan las aplicaciones de Internet por curiosidad y distracción y se identifican con personas de su entorno más cercano, como compañeros de trabajo y personas de su misma edad. Las mujeres por su parte, ven la tecnología como algo positivo que les permite ejercitar la mente, relacionarse, sentirse jóvenes y se identifican con personas que comparten sus mismos intereses sin importar la edad.

En el caso concreto del uso de las redes sociales, ambos desconfían por temor a exponer demasiada información personal. Los hombres las asocian a los jóvenes, por esto las utilizan únicamente con fines profesionales y comerciales. Mientras que las mujeres las usan sólo con fines más personales y afectivos como estar en contacto con familiares y amigos, y se identifican con generaciones más jóvenes. Para ellas el uso de las aplicaciones de Internet ha transformado su vida cotidiana y sienten que pertenecen a la Sociedad de la Información.

\subsection{Búsqueda de información}

Las mujeres buscan información motivadas por simple curiosidad o para ampliar conocimientos y lo 
hacen por Internet porque es una herramienta que permite búsquedas rápidas y cómodas. Los temas son muy variados: Entretenimiento y ocio, política, cultura, salud, historia, nuevas tecnologías, educación, hobbies, noticias...

"Entretenimiento, mapas... (para) afianzarme en lo que estoy aprendiendo [...] en la academia lo aprendo y en casa lo pongo en práctica." (Mujer 4, 63 años)

\section{"Política.” (Mujer 5, 81 años)}

"Viajes, países, enfermedades, cultura, [...] canciones, muchos cuadros..." (Mujer 6, 70 años)

“...busco, por ejemplo, cuando tengo duda de un autor o un compositor o de alguna cuestión histórica... [...] Me resulta más fácil que meterme a una enciclopedia, o porque es muy rápida y porque es cómoda." (Mujer 8, 62 años)

"Bueno, de todo un poquito, si me interesa un tema, sea histórico o sea de alguna persona nombrada que me despertó el interés voy y lo busco o también por temas de estudio y por temas personales... [...] La motivación siempre está, digamos, por el lado que uno quiere ampliar ciertos conocimientos...” (Mujer 2, 52 años)

"Aplicaciones más bien de jardinería, cultura, programas informativos, cosas así [...] (por) curiosidad y por aprender.” (Mujer 3, 66 años)

Algunos hombres consideran que Internet es principalmente una fuente de información sobre diversos temas como política, salud, entretenimiento y ocio, actualidad y otros temas más específicos relacionados con su actividad profesional. El buscador más utilizado es Google.

"Ya le digo, de la política, de lo que pasa cada día, [...] la religión, porque yo soy muy creyente de toda la vida..." (Hombre 5, 81 años) “...mayoritariamente, si mi actividad es del mundo del chocolate, pues intento buscar información sobre el chocolate [...] obviamente voy a Google, ¿quién no va a Google?" (Hombre 10, 61 años)

"Pues todo, depende. Puede ser información de turismo, de viajes, de precios, de compras, de noticias, de películas, de canciones [...] Por curiosidad o el afán de noticias. Para informarme." (Hombre 2, 65 años)

"Para saber exactamente a dónde debo de ir, para no tener ninguna equivocación, ninguna duda en el viaje y cuando llego al sitio, saber lo que veo. Porque ya lo he visto antes." (Hombre 4,76 años)

Tanto hombres como mujeres afirman que no siempre encuentran lo que buscan o tardan en encontrarlo, debido a que la información es excesiva, mala, repetitiva... aunque también reconocen no saber buscar. Por eso recurren a páginas específicas que ya conocen, páginas que comparten su misma ideología o piden ayuda a otras personas:

"Excesiva y muchas veces muy mala." (Mujer 8, 62 años)

"Siempre, pero no como lo querría encontrar. Te vuelvo a repetir que a veces no me aclaran, todos dicen lo mismo, lo mismo, ¡lo mismo!" (Mujer 9, 64 años)

"Más o menos, porque ya veo sitios que sean de tu modo de pensar y claro así ya encuentras más información." (Hombre 5, 81 años)

"Siempre diría que no, quizá porque no lo sé buscar, ¿eh? Pero... otras muchas cosas sí." (Hombre 8, 63 años)

"Mmmmmm... 80\% de veces sí." (Hombre 10, 61 años)

"Siempre. A veces tardo, pero lo encuentro." (Mujer 1, 60 años) 
"Algunas veces sí, y algunas veces pido socorro porque no sé buscarlo bien.” (Mujer 3, 66 años)

Los hombres reciben información de su interés en su correo electrónico porque pertenecen a listas de correos de organizaciones y grupos donde realizan actividades concretas, conforme a su ideología y estilo de vida.

"Porque me mandan a veces mensajes de las situaciones que hay que son de mis ideas y claro, ipues me agrada!" (Hombre 5, 81 años)

"Sólo recibo información de gente a la que conozco porque pertenecemos a organizaciones." (Hombre 8, 63 años)

“...lo uso con amigos y con temas que hago, actividades que desarrollo, algunas de tipo social [...] Tenemos una pequeña organización de mi edad, de matrimonios, básicamente entonces nos dedicamos a hacer labor social, a una congregación católica." (Hombre 9, 61 años)

Hay quien se molesta por la cantidad de información que llega a su correo, que no ha solicitado y que no es de su interés:

"Yo lo que recibo es una cantidad de email basura que no sé, [...] esto la verdad me irrita." (Hombre 10, 61 años)

Las mujeres, también reciben información en su correo electrónico y están suscritas a diferentes listas de correos, pero la variedad de temas por los que se interesan es mucho más amplia: Política, actualidad, ONG's, páginas de facebook, cultura, moda, formación, empleo... Y además, buscan información actualizada en la prensa digital de acuerdo a su ideología.

"No, yo a lo único que estoy inscrita son a los de mi pueblo. O sea, estoy en Cerdanyola al día, y en el momento que hay una reunión en el Ayuntamiento pues ya sabes [...] en seguida me sale en facebook, $[\ldots]$ tengo varias cosas que me mandan así... que días atrás tuve que borrar porque $[\ldots]$ no quiero que me califiquen. Aquí es un pueblo. Todo el pueblo somos amigos..." (Mujer 6, 70 años)

"Recibo boletines electrónicos y los leo...o sea, información acorde a mi vida, a mi estilo de vida." (Mujer 1, 60 años)

"A veces sí, por ejemplo me había inscrito en la Caixa, en un museo que ellos tienen, entonces me llega información de ellos porque yo me había anotado, después de cursos, mucha información de cursos y de empleo también... tengo que tener un correo específico para cada cosa." (Mujer 2, 52 años)

"Recibo correo comercial de tiendas, por ejemplo, recibo de bicing, pero así noticias actualizadas...” (Mujer 9, 64 años)

“...lo que más me gusta es tener información mundial, el periódico, $[\ldots]$ siento un poco la política en mis huesos.” (Mujer 7, 71 años)

En lo respectivo a Búsqueda de información, hombres y mujeres mayores cubren sus necesidades de información al hacer uso de Internet debido a la gran diversidad de fuentes, pero sus intereses son diferentes.

\subsection{Acceso a otros medios}

La mayoría de las mujeres accede a otros medios a través de Internet: Leen el periódico, escuchan la radio o ven programas de TV:

"Para ver TV sí, para periódico también, [...] lo que yo hago es ponerme en el ordenador para ver una cosa que leí, [...] ver las opiniones de otros periódicos de alguna cosa que me interesa, para comparar, [...] pongo música cuando plancho." (Mujer 6, 70 años)

“Sí, para leer el periódico.” (Mujer 7, 71 años)

"Para escuchar radio, sí. Accedo porque sé que hay programas de radio que están por podcast y los busco.” (Mujer 1, 60 años) 
"No, generalmente es para escuchar música." (Mujer 2, 52 años)

"Para leer el periódico, sí." (Mujer 3, 66 años)

Y sólo algunas no lo hacen porque desconocen esta posibilidad o porque prefieren los medios tradicionales:

"Para ver TV no. Pero sí leo el periódico por Internet.” (Mujer 5, 81 años)

“No. ¡Por el portátil no!” (Mujer 4, 63 años)

“¿Para ver TV? No, ahora me entero. ¿Para escuchar la radio? Nooooo, ni sé si existe. (Leer el periódico) Sí, es lo primero que hago." (Mujer 10, 89 años)

"No me gusta nada. No me gusta ver la televisión en Internet, prefiero, ver la televisión en la televisión y ver Internet en Internet. Sí, me parece incómodo lo otro.” (Mujer 8, 62 años)

"Para mí es una desventaja el no tener a una persona a lado para que me ayude. Ahora mismo, tengo problemas para poner contraseña para poder entrar." (Mujer 10, 89 años)

La mayoría de los hombres también hace uso de Internet para acceder a otros medios: Leen diferentes diarios, escuchan la radio, bajan música y ven programas de TV, aunque esto último sólo de manera puntual, ya que prefieren el medio tradicional.

"Sí, para leer el periódico." (Hombre 5, 81 años)

“Ś́lo para leer los periódicos." (Hombre 9, 61 años)

“...para leer periódicos y escuchar el radio." (Hombre 3, 59 años)

"Sí, algunas veces sí. Algunas veces como nosotros colaboramos con programas de radio y no he podido escuchar en directo el programa, entonces accedo a Internet a la hemeroteca y los escucho o los veo...” (Hombre 10, 61 años)

“...para ver TV muy pocas veces.... A veces lo he hecho para las televisiones extranjeras. Aunque se puede hacer en casa de otros amigos, yo no. ¿Radio? Lo hacía, para escuchar radio extranjera, no las de aquí. Para leer periódicos, sí, bastante." (Hombre 2, 65 años)

“No, normalmente la vemos aquí, me gusta más aquí que por Internet. (Señala la TV que tiene en el salón de su casa)” (Hombre 6, 77 años)

"No. A menos que sea TV a la carta. Música y leer periódicos." (Hombre 7, 67 años)

Para la mayoría de hombres y mujeres, las ventajas al hacer uso de Internet son múltiples, como la posibilidad de elegir los contenidos que les interesan y en el momento que lo desean, y esto les anima a usarlo como herramienta para acceder a otros medios:

"Estar actualizada... dentro de mis posibilidades, estar actualizada." (Mujer 1, 60 años)

"Porque así no lo compro y si quiero alguna noticia de mi ciudad lo veo ahí." (Mujer 3, 66 años)

"Comparación, $[\ldots]$ mi hermano me dice, $[\ldots]$ "mira en el periódico tal han puesto esto" que en cambio en La Vanguardia no lo han puesto." (Mujer 6, 70 años)

"Si pierdo algún programa de la TV de noticias, yo lo puedo pillar por Internet." (Mujer 5, 81 años)

"[...] la noticia es inmediata, anterior a la edición del periódico, mañana me meto a Internet y está noche tengo la noticia que aparece en el periódico al día siguiente. La noticia siempre es anticipada en Internet, pero sí algunas noticias, no todo el periódico.” (Hombre 4, 76 años)

“...no me cuesta dinero comprarlo, y que leo 
todos los periódicos que me apetecen, ¡todos los periódicos que quieras! ¿Inconveniente? ninguno. Ventajas, todas, ¡está clarísimo!" (Hombre 5, 81 años)

“...porque es instantáneo, y luego porque en Internet puedes leer 20 periódicos y oirá la opinión o las voces de tantos periódicos diferentes...” (Hombre 8, 63 años)

“... estar al tanto de lo que pasa en México, siendo imposible tener el periódico de papel..." (Hombre 7, 67 años)

"El de México no lo tienes en la mano, entonces ahí lo puedes ver [...] y porque es mucho más fácil ver las noticias del día que bajar comprar el periódico. Subir. Leerlo.” (Hombre 2, 65 años)

Algunos hombres expresaron las desventajas que ven al hacer uso de Internet, sobre todo en la falta de fiabilidad de las fuentes debido al exceso y rapidez de información que impide la concentración y la valoración crítica de la misma:

"La desventaja, supongo es que si estás muy enganchado y si sobre todo si no tienes capacidad de filtro y de valoración crítica de lo que te ponen. Ahí estás perdido entonces." (Hombre 8,63 años)

“¿Desventajas? puede ser que la multiplicidad de fuentes, la rapidez con que se da todo e impide la concentración, [...] y uno le gusta una Ópera y va a YouTube, pero claro hay cuarenta y siete fragmentos repetidos por veintitrés (personas diferentes) y eso lo vuelve loco a uno. (Hombre 2, 65 años)

Además de las actividades mencionadas anteriormente (TV, radio y prensa digital), las mujeres hacen otro uso de Internet:

"Bajo recetas para cocinar por Internet. Yo enciendo el Internet por la mañana y ya sé que está listo, entonces yo, mientras que caliento la plancha voy a Internet. Estoy pillada." (Mujer 6, 70 años)

“¿Importantes? Pues el juicio de Australia, y además estoy metida en la Asociación de la Memoria Histórica de España. [...] el médico forense me dijo que me metiera en facebook...” (Mujer 10, 89 años)

Mientras que los hombres, sobre todo los que siguen activos, hacen uso de aplicaciones más avanzadas de Internet dentro de sus centros de trabajo. Esto les lleva a desarrollar habilidades más complejas, lo que desemboca en el gusto por la tecnología y la adquisición de nuevos dispositivos.

"He creado cursos, diagnósticos psicológicos, comparto fotos, y con el IPhone, todas las aplicaciones, todas las bromas.” (Hombre 7, 67 años)

"Sí, hemos hecho videoconferencias a través de nuestros colegas de Bruselas." (Hombre 10, 61 años)

Al hacerles escoger entre Internet (digital) o cualquier otro medio tradicional (analógico), la mayoría de las mujeres prefiere quedarse con Internet.

"Para mí, me quedaba con el Internet. Ahora bien, pensando en mí marido, la televisión. Internet porque tengo la información enseguida por la mañana." (Mujer 7, 71 años)

"Utilizo más Internet que la TV, lo veo más útil que la TV.” (Mujer 9, 64 años)

“Internet y TV,..." (Mujer 2, 52 años)

"Con Internet, porque avanza más. Es más amplio.” (Mujer 3, 66 años)

“...me quedo con Internet hombre... es mucho mejor. Porque ahí hay diversidad de todo." (Mujer 4, 63 años) 
Y sólo algunas mujeres prefieren la TV, la radio e incluso el teléfono antes que Internet.

“... con la TV.” (Mujer 5, 81 años)

“...no me desprendería nunca del tocadiscos, de la radio, el Internet y luego la televisión, en ese orden.” (Mujer 8, 62 años)

"[...] si me ocurre algo grave, puedo avisar a los médicos $[\ldots]$ está el teléfono también, ¿sabes? Pero urgente, urgente el teléfono.” (Mujer 10, 89 años)

"Con la radio. Porque [...] me gusta escuchar distintos programas y no estoy con la pantalla." (Mujer 1, 60 años)

La mayoría de los hombres, igual que las mujeres, prefieren Internet, ya que les permite acceder a otros medios y no al revés.

"Pues me quedaría con Internet. Es que por Internet haces todo ves la TV, oyes el radio, haces todo por Internet está todo." (Hombre 5, 81 años)

"Con Internet porque me permite todo." (Hombre 7, 67 años)

“Me quedaría con Internet." (Hombre 9, 61 años)

"Y bueno, si existe Internet... prescindiría de todo, menos de Internet." (Hombre 2, 65 años)

Mientras que una minoría prefiere los medios tradicionales, aunque depende de la actividad que quieran realizar.

"Yo siempre me quedaba o bien con la tele o Internet. Me quedaba después con TV, distrae mucho.” (Hombre 6, 77 años)

"Quizá con la televisión porque es más cómodo." (Hombre 1, 55 años)
"Pues casi que me quedaría con Internet, pero vaya... según para que cosas no para todo.”

(Hombre 4, 76 años)

En Acceso a otros medios, hombres y mujeres mayores consideran que podrían prescindir de los medios tradicionales (radio, TV, prensa) ya que saben que se puede acceder a ellos a través de Internet. De hecho, la mayoría lee la prensa digital y escucha música en línea, sin embargo, en el caso concreto de la televisión, siguen utilizando el medio tradicional. De aquí la importancia de que este colectivo desarrolle habilidades digitales debido a esta convergencia de medios.

\subsection{Futuro}

Los hombres (una mayoría), son más pesimistas que las mujeres de cara al futuro: se sienten limitados por la edad, creen que la tecnología no es para ellos y no le sacan partido porque no saben usarla.

"Ninguna, ya estoy caducado yo. Ni idea. Ya no estaré en este mundo [...] Yo con setenta y seis años pocas ganas voy a hacer y me voy a interesar por estas cosas. [...].” (Hombre 4, 76 años)

"Ya poca esperanza, ya poco, con la edad que tengo, ya puedo hacer poco y cuando llegue a esa edad..." (Hombre 6, 77 años)

“... el que sabe, $[\ldots]$ le saca mucho más partido, infinitamente que yo." (Hombre 8, 63 años)

Aunque los más optimistas (una minoría) sí se imaginan haciendo uso de Internet y sus aplicaciones en el futuro e incluso quieren aprender más sobre su manejo.

"Me gustaría saber desarrollar webs, saber informática. [...] ahora ya se pueden ver vídeos y alta definición [...] Desconozco las Redes Sociales, pero creo que es un mundo aparte" (Hombre 6, 77 años)

"Siempre me ha gustado estar más o menos al día (como) perfeccionar el photoshop, lo sé usar y lo uso.” (Hombre 7, 67 años) 
En el caso de las mujeres, la mayoría quiere desarrollar nuevas habilidades digitales y profundizar en las que ya conoce.

"Sobre todo, las de conectarme perfectamente y de saber lo de Internet y todo lo que es de hoy, la informática, todo lo actual.” (Mujer 7, 71 años)

"Bueno, me gustaría saber el manejo de los blogs de poder crear páginas web, a pesar de que no tengo ni idea pero me gustaría." (Mujer 2, 52 años)

"Me gustaría dominar más y poder saber hacer más cosas. No sé, que no tuviera problemas." (Mujer 6, 70 años)

Y sólo una minoría cree que ya ha aprendido lo suficiente, se siente limitada por la edad y no ve la tecnología como algo necesario.

“¿Más?... como gustarme, me gustaría, pero estoy saciada. [...] es más que para la edad que tengo, no, no, yo estoy más tranquila y no necesito nada." (Mujer 8, 62 años)

"No, yo ya no... ya tengo bastante [...] es muy problemático, yo que sé mi futuro cuál es. No le veo futuro... he de contar con que tengo ochenta y dos años, no sé, mi futuro es a corto plazo.” (Mujer 5, 81 años)
Ambos temen depender de la tecnología y perder el trato personal.

"Internet, según anuncian te va a permitir hacer todo, ¿Qué pasa?, ¿qué no vamos a salir de casa? Se está perdiendo el contacto físico, ahora vamos a comprar por Internet un ordenador y te lo van a enviar [...]" (Hombre 3, 59 años)

"Mi vida, yo creo que será igual. No me voy a transformar por mejorar esto. Lo utilizo como hobby y como fuente de conocimiento y para estudiar, pero nada más." (Hombre 9, 61 años)

"Mi vida creo que va a continuar más o menos de esta forma, porque tengo una disciplina para el uso de Internet, porque dentro del ocio, no me conecto a Internet si no que busco la naturaleza." (Mujer 1, 60 años)

"(cambiaría) Mucho y de forma positiva pero siempre guardando mi creatividad, no que me absorba.” (Mujer 2, 52 años)

Y por último, el tópico Futuro, en el que se detectó que hombres y mujeres mayores "temen" el cambio tecnológico, porque no son capaces de imaginar el alcance que puede tener debido a la rapidez de los avances y al mismo tiempo no quieren depender de la tecnología.

\section{Diario de campo}

Como anteriormente se ha mencionado, el diario de campo fue un complemento a la entrevista. Con este instrumento se pretendía realizar una comparación entre hombres y mujeres mayores al hacer uso de Internet para saber cuáles son las aplicaciones y actividades que realizan en línea y de esta manera indagar sobre cuáles son las habilidades digitales que poseen y las necesidades que cubren con el uso de las mismas.

Encontramos muchas ventajas al introducir el diario de campo ya que es una herramienta de gran utili- dad para conocer el tiempo de uso y/o las dificultades que pueden surgir al hacer uso de cada una de las aplicaciones de Internet. Además de proporcionar información de primera mano.

Como limitación encontramos que el diario de campo, al ser un instrumento que debían llevarse a casa para registrar sus actividades en línea, fue difícil de recuperar. De los veinte diarios de campo entregados sólo fueron devueltos diez de ellos, correspondientes a seis mujeres y cuatro hombres. 
A pesar del seguimiento realizado por teléfono y correo electrónico para recuperar los diarios al final esto no fue posible. Algunos sujetos que sí lo devolvieron, comentaron que llevar el registro de sus actividades en línea parecía una obligación, una tarea más que realizar en su día a día.

De aquí que los resultados de la muestra hayan quedado incompletos y mucha de la información no se vea reflejada.

Por esto, se considerada que para próximos estudios se debería ofrecer una recompensa, ya sea monetaria o un pequeño obsequio, para que los sujetos muestren más interés y se sientan comprometidos con el estudio en el que participan. A pesar de todo, se ha estimado conveniente dar a conocer estos resultados.

El gráfico que a continuación se presenta muestra el tiempo que dedican hombres y mujeres mayores al uso de las aplicaciones de Internet durante diez días (ver gráfico 2).

\section{Gráfico 2: Tiempo de uso de las aplicaciones de Internet en hombres y mujeres mayores.} Diario de campo de 10 días.

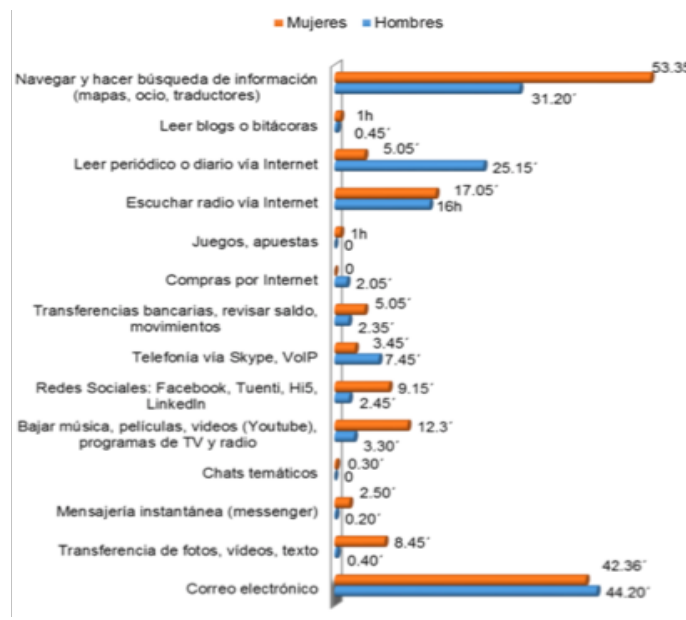

Fuente: Elaboración propia
Las mujeres pasan más tiempo frente al ordenador navegando por la red y buscando información que los hombres (53.35' - 31.20'), usan más las Redes Sociales $\left(9.15-2.45^{\prime}\right)$, utilizan con más frecuencia aplicaciones para bajar música, películas y vídeos $\left(12.30^{\prime}-3.30^{\prime}\right)$ y realizan transferencias de fotos, vídeos y texto $\left(8.45^{\prime}-0.40^{\prime}\right)$.

Los hombres, por el contrario, dedican más tiempo a leer el periódico vía Internet $\left(25.15^{\prime}-5.05^{\prime}\right)$ y al uso de la telefonía vía Skype o VoIP $\left(7.45^{\prime}-3.45^{\prime}\right)$.

Ambos dedican gran parte de su tiempo a escuchar la radio $\left(16-17.05^{\prime}\right)$ y al uso del correo electrónico $\left(44.20^{\prime}-42.36^{\prime}\right)$.

Las aplicaciones no utilizadas por hombres y mujeres son los grupos de discusión en línea, el pago de servicios y la TV a través de Internet.

En resumen, podemos afirmar que tanto hombres como mujeres mayores emplean la mayor parte del tiempo en leer y enviar correos electrónicos, navegar por la red y leer el periódico vía Internet.

Sin embargo, es muy poco el tiempo de uso que dedican a aplicaciones como chats temáticos, blogs y bitácoras, aunque el tiempo de uso en las mujeres es ligeramente mayor al tiempo de uso que dedican los hombres.

Esto demuestra que el uso de las aplicaciones y actividades antes mencionadas, responde a las necesidades que hombres y mujeres mayores cubren al hacer uso de la tecnología. 


\section{Discusión}

El hecho de que las personas mayores prefieran comenzar a hacer uso de la tecnología en lugares públicos como bibliotecas, responde en gran medida a la sensación de miedo al no saber usar un ordenador y la seguridad que les aporta tener a alguien cerca que los auxilie en caso necesario. En nuestro estudio, la ayuda de generaciones más jóvenes contribuye a motivar a este colectivo a hacer uso de la tecnología y desarrollar las habilidades digitales necesarias para cubrir algunas de sus necesidades.

Lewis y Menn (2009) expresan que los ordenadores se han convertido en un bien imprescindible para muchas personas. Y que en el caso de las personas mayores, antes de adquirir un ordenador prefieren hacer uso de uno donde haya personas que los puedan ayudar a realizar sus actividades en línea.

Ramírez Pino (2008) concluye en su tesis doctoral, que las personas mayores ahora forman parte del cambio social dentro del cambio tecnológico, dejando atrás la asociación tecnología-juventud. Y en este estudio vemos además que las personas mayores quieren pertenecer a la Sociedad de la Información y no quedar en el atraso tecnológico. Es decir, que existe un creciente interés por hacer uso de la tecnología e incorporarla cada vez más a su vida cotidiana.
En el estudio realizado por Bross y Roe (2005) se encuentran algunas diferencias entre hombres y mujeres al usar las aplicaciones de Internet, al demostrar que realmente no existía una brecha digital entre sexos ni una brecha de género, más bien, que el factor más importante es la experiencia en el uso del ordenador debido a que ésta disminuye la ansiedad y la actitud negativa hacia el uso del mismo.

En este estudio se encuentra que efectivamente existe un interés personal por hacer uso de la tecnología sin importar el género de las personas. Sin embargo, son más las mujeres que están interesadas en aprender nuevas habilidades digitales para poder compartir experiencias digitales con sus nietos y no romper la comunicación con generaciones más jóvenes a la suya lo cual las lleva a practicar y desarrollar nuevas habilidades digitales de cara al futuro.

Dato que también se confirma con lo que expresa el Instituto de Mayores y Servicios Sociales (IMSERSO):

"En cierto modo, se reproducen las redes previas a la edad de jubilación, los hombres más próximos a amigos y compañeros del trabajo, y las mujeres más cercanas al mantenimiento y los lazos familiares" (IMSERSO. Libro Blanco del Envejecimiento Activo, 2011: 65).

\section{Conclusiones}

Resumiendo, las aplicaciones de Internet gratifican por igual a hombres y mujeres. Ambos buscan actividades distintas a las que desempeñan habitualmente; las mujeres disfrutan más de las relaciones personales ya que buscan la interacción con los demás, mientras que los hombres optan por actividades más individuales. Los intereses de las mujeres son más amplios y variados, y quieren descubrir las posibilidades que les ofrece la tecnología, mientras que los intereses de los hombres son más limitados. Para ellas el uso de las aplicaciones de Internet ha transformado su vida cotidiana y sienten que pertenecen a la Sociedad de la Información. La gran cantidad y variedad de información se ajusta a los intereses y necesidades de ambos. Saben que se puede acceder a otros medios a través de Internet, y que esto implica la adquisición de nuevas habilidades digitales, aunque en casos concretos como el de la televisión siguen utilizando el medio tradicional. A pesar de que ambos "temen" la rapidez con la que se producen los cambios y el alcance que estos puedan tener, en las mujeres hay un interés por seguir aprendiendo y desarrollando nuevas habilidades de cara al futuro, a diferencia de los hombres que optan por conservar y practicar las habilidades que poseen pero no están interesados en adquirir otras nuevas.

Para futuras investigaciones se propone un estudio cualitativo a través de la entrevista a profundidad que 
recoja las experiencias digitales compartidas de hombres y mujeres mayores con generaciones más jóvenes.

Otra propuesta sería un estudio cualitativo con la misma técnica de recolección de datos dirigida a personas mayores con más experiencia en el uso de la tecnología y que colaboran para que personas de su misma generación, pero con menos experiencia, desarrollen más habilidades digitales.

Finalmente se propone que la perspectiva de género se aplique en ambos estudios.

\section{Referencias}

- Abbate, J. (2000). Inventing the internet. MIT press. En Castells, Manuel. (2000) La era de la información: Economía, sociedad y cultura. Vol. 3: Fin de milenio. Edit. Alianza. Madrid.

- Anderson, Meredith, Wesley, Shrum. (2007) "Circumvention and Social Change: ICT and the Discourse of the Empowerment". Women Studies in Communication. Vol. 30, Number 2, Summer 2007.

- Broos, A. y Roe, K. (2005). "Marginality in the information age: Is the gender gap really diminishing?" Communications: The European Journal of Communication Research, 30(2), 251 260.

- Castells, Manuel. (2004) La sociedad Red: una visión global. Alianza Editorial. Madrid.

- Comisión Europea. Economic and Financial affairs. (2012).The 2012 Ageing Report: Economic and budgetary projections for the $27 \mathrm{EU}$ Member States (2010-2060). [En línea]: (http:/ / ec.europa.eu/economy_finance/publi cations/european_economy/2012/pdf/ee2012-2_en.pdf) [PDF].

- Giddens, Anthony. (1991) Sociología. Alianza Editorial, S.A., Madrid.

- Guba, E. y Lincoln, Y. (2002). "Paradigmas en competencia en la investigación cualitativa”. En Denman, C. y J.A. HARO (Comps.) Por los rincones. Antología de métodos cualitativos en la investigación social. El Colegio de Sonora. Hermosillo, Sonora. 2002, pp. 113-145.
- Instituto de Mayores y Servicios Sociales (IMSERSO), (2011). PDF. Libro blanco del Envejecimiento Activo. [En línea]: <http://www.imserso.es/InterPresent2/gro ups/imserso/documents/binario/8088_8089l ibroblancoenv.pdf $>$ [PDF]

- Katz, E., \& Blumler, J. G. (1974). The uses of mass communications: Current perspectives on gratifications research. Beverly Hills, Calif.: Sage.

- Krippendorff, K. (1990). Metodología del análisis de contenido. Teoría y Práctica. Barcelona. Paidós Ibérica. S.A.

- Lewis, Clayton y Menn, Lise. (2009) Access Tool? Accelerating Treadmill? Technology and the Aging Population. Institute of Cognitive Science, University of Colorado, Boulder, CO, USA. En Universal Access in Human-Computer Interaction. Addressing Diversity. Book Series: Lecture Notes in Computer Science. Volume 5614/2009. C. Stephanidis (Ed.): Universal Access in HCI, Part I, HCII 2009, LNCS 5614, pp. 263-268, 2009.

- Mcquail, D.; Blumler, J. \& Brown, R. (1972): "The television audience: a revised perspective”. En McQuail, D. (ed.): Sociology of Mass Communication. London: Longman.

- Mordini, Emilio; Wright, David; Wadhwa, Kush; De Hert, Paul; Mantovani, Eugenio; Thestrup, Jesper; Van Steendam, Guido; D'Amico, Antonio and Vater, Ira. (2009) "Senior citizens and the ethics of e-inclusion" Ethics and Information Technology, Volume 11, Number 3 / September, 2009. 
- Pike, Kenneth L. (1954). Language in relation to a unified theory of the structure of human behavior. Dallas, TX, US: Summer Institute of Linguistics. (1954).

- PNUD, Programa de las Naciones Unidad para el Desarrollo. Informe de desarrollo Humano. (2001). Poner el Desarrollo Tecnológico al Servicio del Desarrollo Humano. [En línea]: (http://hdr.undp.org/en/media/HDR_2001 _ES.pdf) [PDF]

- Prensky; M. (2001) "Digital Natives, Digital Immigrants". On the Horizon, NCB University Press, Vol. 9 No. 5, October 2001. En Museos para la Generación E. María Soledad Gómez Vílchez. [En línea]:

$<$ http://mediamusea.files.wordpress.com/20 07/11/museos-para-la-generacion-e.pdf, http://mediamusea.com>

- Ramírez Pino, Rodrigo (2008). ¿Dónde estás? La telefonía móvil y la vida cotidiana. El caso de las personas mayores en Barcelona. Dir.: Rosa Franquet. (Tesis Doctoral)

- Sampieri Hernández, Roberto. (1997, 2007). Metodología de la investigación. Madrid. McGraw-Hill.

- Strauss, A., \& Corbin, J. (2002). Bases de la investigación cualitativa: técnicas y procedimientos para desarrollar la teoría fundamentada. Medellín: Universidad de Antioquia.

- Vázquez, Susana y Castaño, Cecilia. (2011). "La brecha digital de género: prácticas de einclusión y razones de la exclusión de las mujeres”. Asparkía. Investigación feminista. No. 22, 33-49.

- Zamorano, José Luis; Gil-Loyzaga, Pablo y Maravet, Dolores (coords.) (2004) Telemedicina. Análisis de la situación actual y perspectivas del futuro. Edita Fundación VODAFONE.

\section{Forma de Citación}

ÁLVAREZ-ARÁMBULA, Erika Guadalupe: Uso de las aplicaciones de Internet en personas mayores en Barcelona: Un estudio desde la perspectiva de género. Revista Communication Papers, $\mathrm{N}^{\circ} 7$, páginas 124 a 140 . Departamento de Filología y Comunicación de la Universidad de Girona. Recuperado el __ de de 2 de: http: / / www.communicationpapers.es 